\title{
Comments on 'Area and power efficient DCT architecture for image compression' by Dhandapani and Ramachandran
}

Renato J. Cintra ${ }^{1 *}$ and Fábio M. Bayer ${ }^{2}$

\begin{abstract}
In [Dhandapani and Ramachandran, "Area and power efficient DCT architecture for image compression", EURASIP Journal on Advances in Signal Processing 2014, 2014:180] the authors claim to have introduced an approximation for the discrete cosine transform capable of outperforming several well-known approximations in literature in terms of additive complexity. We could not verify the above results and we offer corrections for their work.
\end{abstract}

Keywords: DCT approximations, Low-complexity transforms

\section{Introduction}

In a recent paper [1], a low-complexity transformation was introduced, which is claimed to be a good approximation to the discrete cosine transform (DCT). We wish to evaluate this claim.

The introduced transformation is given by the following matrix:

$$
\mathbf{T}=\left[\begin{array}{cccccccc}
1 & 0 & 0 & 0 & 0 & 0 & 0 & 1 \\
1 & 1 & 0 & 0 & 0 & 0 & 1 & 1 \\
0 & 0 & 1 & 0 & 0 & 1 & 0 & 0 \\
0 & 0 & 1 & 1 & 1 & 1 & 0 & 0 \\
0 & 0 & 1 & 1 & -1 & -1 & 0 & 0 \\
0 & 0 & 1 & 0 & 0 & -1 & 0 & 0 \\
1 & 1 & 0 & 0 & 0 & 0 & -1 & -1 \\
1 & 0 & 0 & 0 & 0 & 0 & 0 & -1
\end{array}\right]
$$

We aim at analyzing the above matrix and showing that it does not consist of a meaningful approximation for the 8-point DCT. In the following, we adopted the same methodology described in [2-11] which the authors also claim to employ.

${ }^{*}$ Correspondence: rjdsc@stat.ufpe.org

'Departmento de Estatística, Universidade Federal de Pernambuco, Recife, Brazil

Full list of author information is available at the end of the article

\section{Criticisms}

\subsection{Inverse transformation}

The authors of [1] claim that inverse transformation $\mathbf{T}^{-1}$ is given by

$$
\frac{1}{2} \cdot\left[\begin{array}{cccccccc}
1 & 0 & 0 & 0 & 1 & 0 & 0 & 0 \\
-1 & 1 & 0 & 0 & -1 & 1 & 0 & 0 \\
0 & 0 & 1 & 0 & 0 & 0 & 1 & 0 \\
0 & 0 & -1 & 1 & 0 & 0 & -1 & 1 \\
0 & 0 & -1 & 1 & 0 & 0 & 1 & -1 \\
0 & 0 & 1 & 0 & 0 & 0 & -1 & 0 \\
-1 & 1 & 0 & 0 & 1 & -1 & 0 & 0 \\
1 & 0 & 0 & 0 & -1 & 0 & 0 & 0
\end{array}\right] .
$$

However, simple computation reveal that this is not accurate, being the actual inverse given by:

$$
\mathbf{T}^{-1}=\frac{1}{2} \cdot\left[\begin{array}{cccccccc}
1 & 0 & 0 & 0 & 0 & 0 & 0 & 1 \\
-1 & 1 & 0 & 0 & 0 & 0 & 1 & -1 \\
0 & 0 & 1 & 0 & 0 & 1 & 0 & 0 \\
0 & 0 & -1 & 1 & 1 & -1 & 0 & 0 \\
0 & 0 & -1 & 1 & -1 & 1 & 0 & 0 \\
0 & 0 & 1 & 0 & 0 & -1 & 0 & 0 \\
-1 & 1 & 0 & 0 & 0 & 0 & -1 & 1 \\
1 & 0 & 0 & 0 & 0 & 0 & 0 & -1
\end{array}\right]
$$

\subsection{Lack of DC component}

The first point to be noticed is that the matrix $\mathbf{T}$ lacks a row of constant entries. Therefore, it is not capable of 


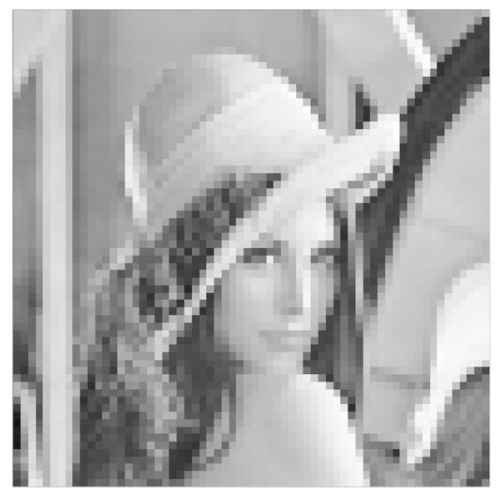

(a) Only DC term (PSNR $=23.66 \mathrm{~dB})$ (b) DCT without DC term

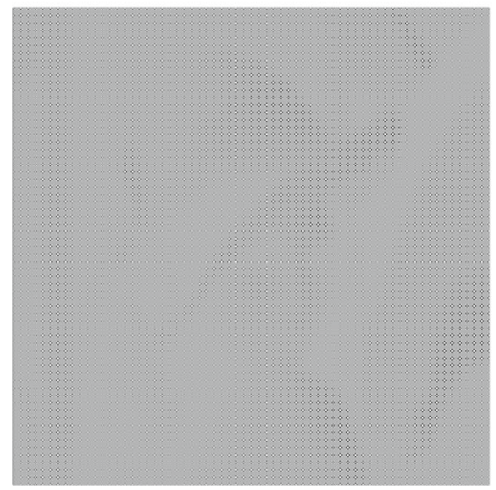

(c) Only first row of $\mathbf{T}[1]$ $(\mathrm{PSNR}=4.70 \mathrm{~dB})$
$(\mathrm{PSNR}=5.67 \mathrm{~dB})$
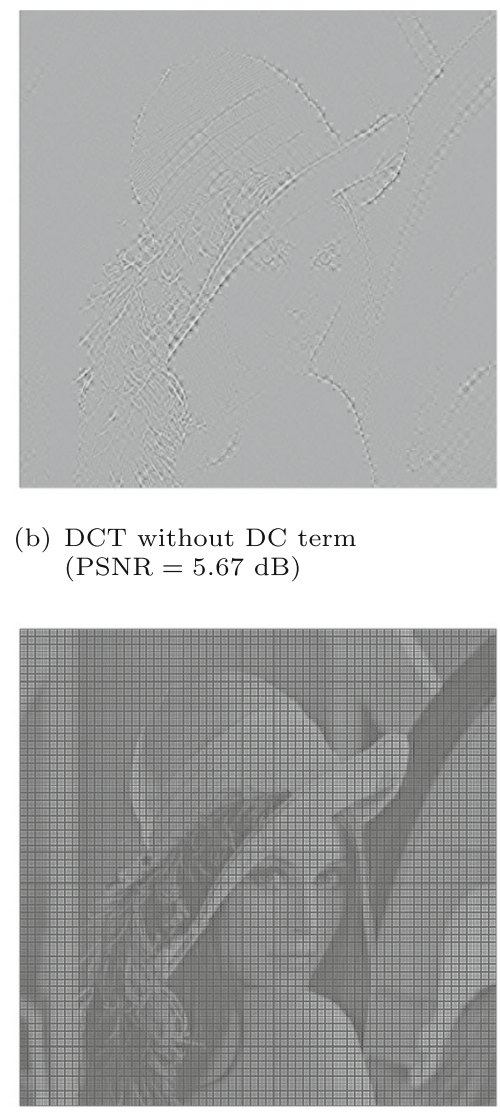

(d) $\mathbf{T}$ without the first row $(\mathrm{PSNR}=4.74 \mathrm{~dB})$

Fig. 1 Reconstructed Lena image based $\mathbf{a}$ only on the DC component of the DCT, $\mathbf{b}$ on all DCT transform coefficient except the DC component, c only on the first row of the $\mathbf{T}[1]$, and $\mathbf{d}$ on all $\mathbf{T}$ coefficients, except the first row

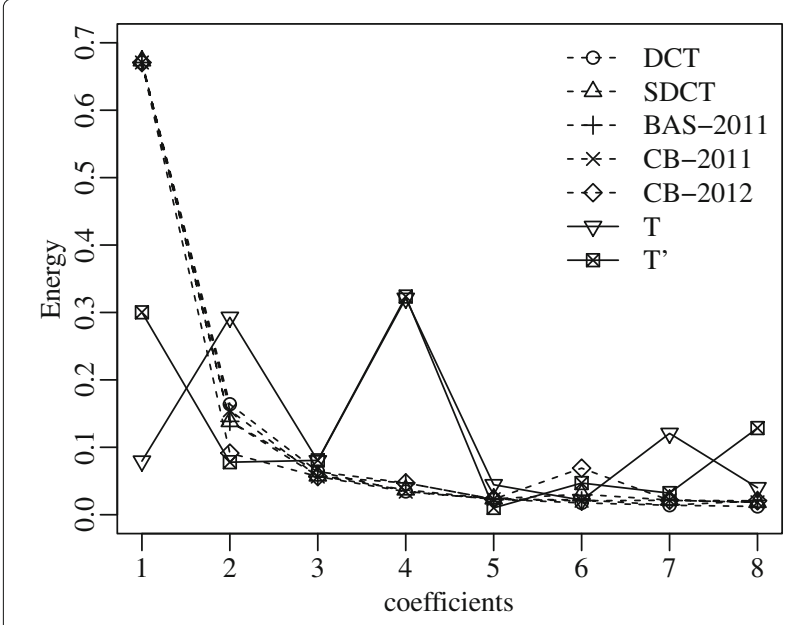

Fig. 2 Energy distribution of transform coefficients
Table 1 Transform coding assessment

\begin{tabular}{lll}
\hline Method & Transform efficiency & Coding gain (dB) \\
\hline DCT [12] & 93.99 & 8.83 \\
SDCT [2] & 82.62 & 6.02 \\
BAS-2008 [4] & 84.95 & 6.01 \\
BAS-2009 [6] & 85.38 & 7.91 \\
BAS-2010 [8] & 88.22 & 8.33 \\
BAS-2011 [9] & 85.38 & 7.91 \\
CB-2011 [13] & 87.43 & 8.18 \\
CB-2012 [3] & 80.90 & 7.33 \\
Transformation in [14] & $\mathbf{3 4 . 9 3}$ & $\mathbf{- 1 . 6 5}$ \\
Transformation in [15] & $\mathbf{3 3 . 6 7}$ & $\mathbf{- 4 . 0 8}$ \\
T [1] & $\mathbf{2 8 . 9 5}$ & $\mathbf{- 1 . 8 6}$ \\
T $_{\text {SFG [1] }}$ & $\mathbf{2 8 . 9 5}$ & $\mathbf{- 1 . 8 6}$ \\
\hline
\end{tabular}


Table 2 PSNR of reconstructed images $(r=6 w 0)$

\begin{tabular}{|c|c|c|c|c|c|c|c|}
\hline Transform & Lena & Boat & Goldhill & Barbara & Lighthouse & Mandrill & Grass \\
\hline DCT [12] & 51.400 & 46.531 & 49.497 & 47.097 & 49.719 & 41.147 & 44.264 \\
\hline SDCT [2] & 45.708 & 41.593 & 44.308 & 40.532 & 43.044 & 35.956 & 36.517 \\
\hline BAS-2008 [4] & 43.996 & 39.498 & 42.449 & 38.304 & 41.139 & 33.886 & 34.364 \\
\hline BAS-2009 [6] & 48.096 & 44.828 & 46.470 & 40.143 & 44.035 & 37.982 & 36.869 \\
\hline BAS-2010 [8] & 50.976 & 46.483 & 48.912 & 46.657 & 48.193 & 40.617 & 42.486 \\
\hline BAS-2011 [9] & 48.010 & 44.874 & 46.328 & 40.073 & 44.690 & 38.085 & 37.191 \\
\hline CB-2011 [13] & 49.537 & 45.353 & 47.892 & 43.163 & 46.455 & 39.668 & 39.815 \\
\hline CB-2012 [3] & 46.621 & 44.217 & 45.027 & 39.763 & 41.939 & 36.486 & 35.223 \\
\hline Transformation in [14] & 30.193 & 29.635 & 32.107 & 29.411 & 29.777 & 26.575 & 20.612 \\
\hline Transformation in [15] & 27.895 & 27.463 & 29.797 & 27.260 & 27.547 & 24.530 & 18.445 \\
\hline $\mathbf{T}[1]$ & 30.560 & 30.034 & 32.565 & 29.851 & 30.090 & 26.862 & 20.982 \\
\hline $\mathbf{T}_{\mathrm{SFG}}[1]$ & 30.889 & 29.867 & 32.920 & 29.117 & 30.189 & 26.779 & 20.900 \\
\hline
\end{tabular}

computing the mean value or the DC component of a signal under analysis. In terms of image compression, the DC value is the single most important coefficient concentrating most of the image energy. To illustrate this fact, Fig. 1 shows the reconstructed standard Lena image by means of (i) the DC component of the standard DCT, (ii) all DCT coefficients, except the DC component, (iii) the first row of matrix $\mathbf{T}$ [1], and (iv) all $\mathbf{T}$ coefficients, except the first row, respectively. In [12], Britanak meticulously cataloged dozens of DCT approximations; all of them computed the DC component. The lack of the DC component computation suggests that compressed images resulting from the application of $\mathbf{T}$ are expected to be severely degraded in terms of perceived image quality. The associated PSNR values in Fig. 1 also show the poor quality of the reconstructed images using $\mathbf{T}$. Considering $M \times N$ pixel images, the PSNR measure is calculated by:

$$
\operatorname{PSNR}=10 \cdot \log _{10}\left(\frac{\mathrm{MAX}^{2}}{\mathrm{MSE}}\right)
$$

where MSE $=\frac{1}{M \cdot N} \sum_{i=1}^{M} \sum_{j=1}^{N}\left(a_{i, j}-b_{i, j}\right)^{2}, a_{i, j}$ and $b_{i, j}$ are the $(i, j)$-th element of the original and reconstructed images, respectively; and MAX is the maximum pixel valye. For 8-bit greyscale images, $\mathrm{MAX}=255$.

\subsection{Fast algorithm}

In the 'Fig. 1' of [1], the authors display a signal flow graph (SFG) which does not correspond to the computation implied by their proposed matrix. Their proposed SFG consists of two addition butterfly sections and one final permutation, which correspond to the following matrices, respectively:

$$
\mathbf{A}_{1}=\left[\begin{array}{cccccccc}
1 & 0 & 0 & 0 & 0 & 0 & 0 & -1 \\
0 & 1 & 0 & 0 & 0 & 0 & -1 & 0 \\
0 & 0 & 1 & 0 & 0 & -1 & 0 & 0 \\
0 & 0 & 0 & 1 & -1 & 0 & 0 & 0 \\
0 & 0 & 0 & 1 & 1 & 0 & 0 & 0 \\
0 & 0 & 1 & 0 & 0 & 1 & 0 & 0 \\
0 & 1 & 0 & 0 & 0 & 0 & 1 & 0 \\
1 & 0 & 0 & 0 & 0 & 0 & 0 & 1
\end{array}\right]
$$

\begin{tabular}{|c|c|c|c|c|c|c|c|}
\hline Transform & Lena & Boat & Goldhill & Barbara & Lighthouse & Mandrill & Grass \\
\hline DCT [12] & 32.088 & 28.971 & 30.656 & 24.752 & 25.549 & 22.832 & 19.893 \\
\hline SDCT [2] & 27.443 & 25.570 & 27.543 & 23.488 & 23.348 & 21.095 & 17.019 \\
\hline BAS-2008 [4] & 29.509 & 27.150 & 28.994 & 24.285 & 24.444 & 22.279 & 18.849 \\
\hline BAS-2009 [6] & 29.916 & 27.354 & 29.288 & 24.520 & 24.381 & 22.223 & 18.661 \\
\hline BAS-2010 [8] & 31.143 & 28.292 & 30.072 & 24.666 & 25.063 & 22.581 & 19.376 \\
\hline BAS-2011 [9] & 29.916 & 27.354 & 29.288 & 24.520 & 24.381 & 22.223 & 18.661 \\
\hline CB-2011 [13] & 30.446 & 27.861 & 29.612 & 24.460 & 24.756 & 22.516 & 19.157 \\
\hline CB-2012 [3] & 27.015 & 25.190 & 27.141 & 23.595 & 23.087 & 21.596 & 17.170 \\
\hline Transformation in [14] & 2.159 & 1.856 & 2.877 & 2.936 & 2.582 & 1.992 & 1.981 \\
\hline Transformation in [15] & -6.927 & -7.213 & -6.205 & -6.120 & -6.442 & -7.053 & -6.914 \\
\hline $\mathbf{T}[1]$ & 2.163 & 1.867 & 2.880 & 2.951 & 2.596 & 2.001 & 1.981 \\
\hline $\mathbf{T}_{\mathrm{SFG}}[1]$ & 4.686 & 4.380 & 5.399 & 5.454 & 5.086 & 4.481 & 4.355 \\
\hline
\end{tabular}

Table 3 PSNR of reconstructed images $(r=10)$ 


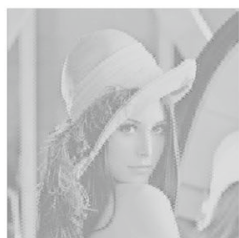

(a)

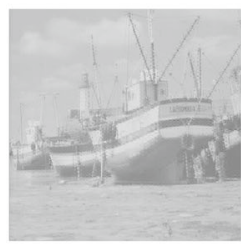

(e)

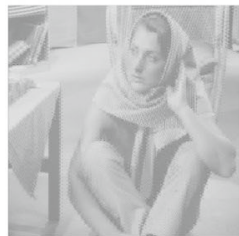

(i)

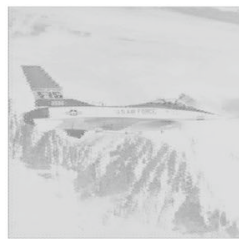

(m)

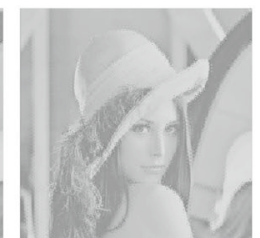

(b)

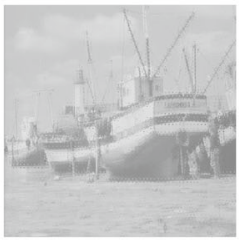

(f)

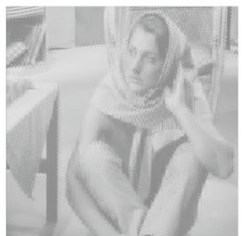

(j)

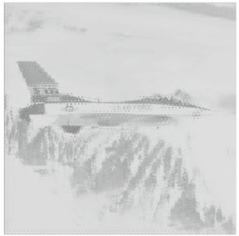

(n)

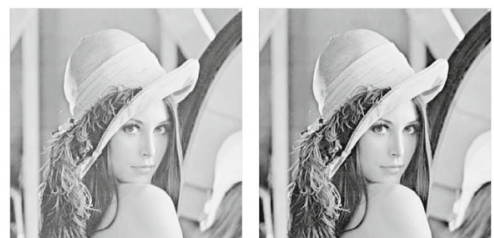

(c)

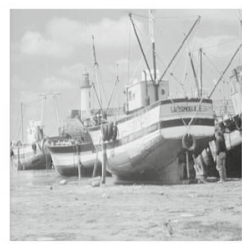

(g)

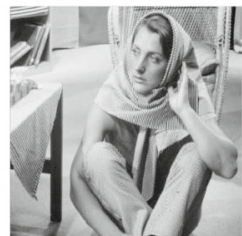

$(\mathrm{k})$

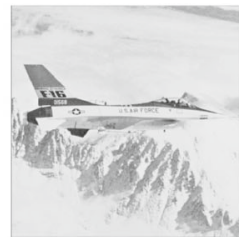

(o) (d)

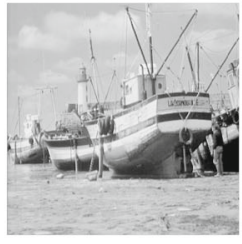

(h)

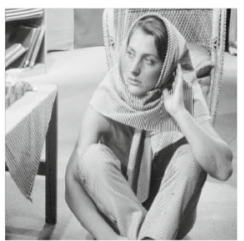

(1)

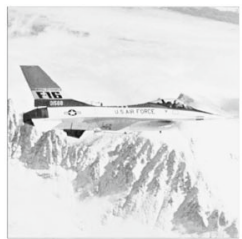

(p)

Fig. 3 Reconstructed images for $r=45$. a T (PSNR=23.743), b TSFG (PSNR=24.244), c CB-2012 (PSNR=36.977), d BAS-2011 (PSNR=39.835),

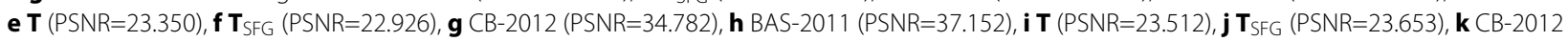
$(P S N R=29.913), \mathbf{I} B A S-2011$ (PSNR=31.298), $\mathbf{m} \mathbf{T}(P S N R=22.282), \mathbf{n} \mathbf{T}_{\mathrm{SFG}}(\mathrm{PSNR}=22.184), \mathbf{o} \mathrm{CB}-2012(\mathrm{PSNR}=36.785)$ and $\mathbf{p} B A S-2011(\mathrm{PSNR}=40.433)$

$\mathbf{A}_{2}=\left[\begin{array}{llllllll}1 & 1 & 0 & 0 & 0 & 0 & 0 & 0 \\ 0 & 1 & 0 & 0 & 0 & 0 & 0 & 0 \\ 0 & 0 & 1 & 1 & 0 & 0 & 0 & 0 \\ 0 & 0 & 0 & 1 & 0 & 0 & 0 & 0 \\ 0 & 0 & 0 & 0 & 1 & 1 & 0 & 0 \\ 0 & 0 & 0 & 0 & 0 & 1 & 0 & 0 \\ 0 & 0 & 0 & 0 & 0 & 0 & 1 & 0 \\ 0 & 0 & 0 & 0 & 0 & 0 & 1 & 1\end{array}\right], \mathbf{P}=\left[\begin{array}{llllllll}0 & 0 & 0 & 0 & 0 & 0 & 0 & 1 \\ 0 & 0 & 0 & 0 & 0 & 0 & 1 & 0 \\ 0 & 0 & 0 & 0 & 0 & 1 & 0 & 0 \\ 0 & 0 & 0 & 0 & 1 & 0 & 0 & 0 \\ 0 & 0 & 0 & 1 & 0 & 0 & 0 & 0 \\ 0 & 0 & 1 & 0 & 0 & 0 & 0 & 0 \\ 0 & 1 & 0 & 0 & 0 & 0 & 0 & 0 \\ 1 & 0 & 0 & 0 & 0 & 0 & 0 & 0\end{array}\right]$

However, this fast algorithm induces to the following matrix:

$\mathbf{P} \cdot \mathbf{A}_{2} \cdot \mathbf{A}_{1}=\left[\begin{array}{cccccccc}1 & 1 & 0 & 0 & 0 & 0 & 1 & 1 \\ 0 & 1 & 0 & 0 & 0 & 0 & 1 & 0 \\ 0 & 0 & 1 & 0 & 0 & 1 & 0 & 0 \\ 0 & 0 & 1 & 1 & 1 & 1 & 0 & 0 \\ 0 & 0 & 0 & 1 & -1 & 0 & 0 & 0 \\ 0 & 0 & 1 & 1 & -1 & -1 & 0 & 0 \\ 0 & 1 & 0 & 0 & 0 & 0 & -1 & 0 \\ 1 & 1 & 0 & 0 & 0 & 0 & -1 & -1\end{array}\right]=\mathbf{T}_{\mathrm{SFG}}$ which is different from $\mathbf{T}$. Therefore, the SFG is incorrect and does not correspond to the proposed matrix. We assume that the intended method is $\mathbf{T}_{\mathrm{SFG}}$, which is the matrix implied by the fast algorithm. Indeed, this transformation is shown again in the schematics of the hardware realization of their work. Nevertheless, hereafter, we analyze both matrices: $\mathbf{T}$ and $\mathbf{T}_{\mathrm{SFG}}$. Similar to $\mathbf{T}$, the matrix $\mathbf{T}_{\mathrm{SFG}}$ does not evaluate the DC value, being subject to the criticism detailed in the previous subsection.

\subsection{Lack of energy concentration}

Contrary to the expected behavior for a data compression transformation, the matrix $\mathbf{T}$ does not exhibit good decorrelation and energy concentration properties. Energy concentration can be quantified by submitting data to a considered transformation and then computing the energy distribution along transform-domain coefficients. Thus, we considered a Monte Carlo simulation with 10,000 8-point input vectors modeled after the firstorder Markov process with correlation coefficient of 0.95 [12]. For comparison, we considered the following 
transformations: the DCT [12], the SDCT [2] the BAS2013 [9], and the BC-2012 [3]. Obtained mean values are displayed in Fig. 2. In clear contrast with the other methods, transformations $\mathbf{T}$ and $\mathbf{T}_{\mathrm{SFG}}$ perform very poorly.

Moreover, the lack of energy concentration in the first transform coefficients indicates that the standard zigzag pattern employed in the quantization step is not adequate for this transformation. Nevertheless, the authors claim to employ the zigzag pattern with success. We could not verify this claim.

To further assess the claim of good coding capabilities, we considered the unified coding gain and the transform efficiency as measures to quantify the coding performance [12] of $\mathbf{T}$ in comparison with bona fide transforms, such as: DCT, SDCT [2], BAS-2008 [4], BAS-2009 [6], BAS-2010 [8], BAS-2011 [9], CB-2011 [13], and CB-2012
[3]. In addition, we also considered the transformation in [14] and transformation in [15]. Results are shown in Table 1. We emphasize in bold the unfavorable measurements associated to the transformations proposed by the authors. Such transformations are not expected to be suitable for image compression, since both coding measures resulted in very low values.

\subsection{Irreproducibility of results}

The results shown by Dhandapani and Ramachandran could not be repeated. The authors state that they employ simultaneously a quantization step, which corresponds to variable bitrate encoding, and a fixed number of retained transform-domain coefficients, which suggests constant bitrate. This seems contradictory. However, to examine the transformation suggested by the authors, we adopted

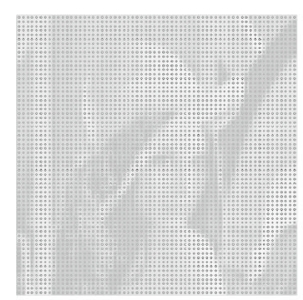

(a)

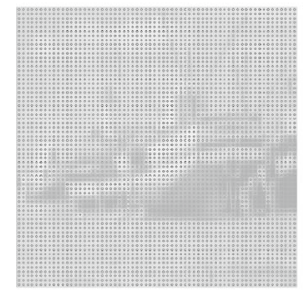

(e)

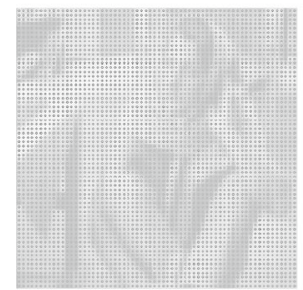

(i)

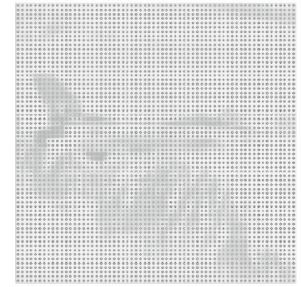

$(\mathrm{m})$

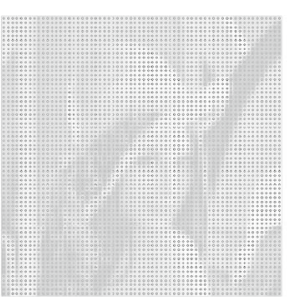

(b)

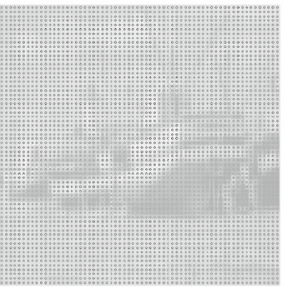

(f)

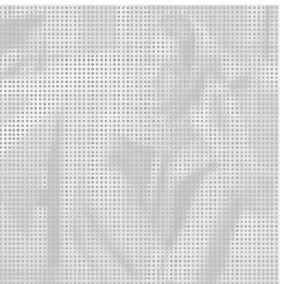

(j)

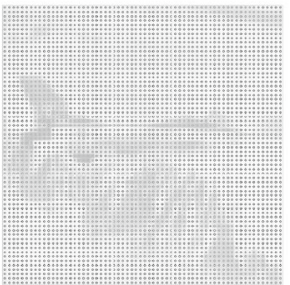

(n)

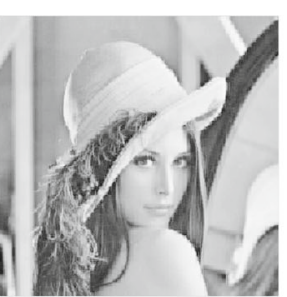

(c)

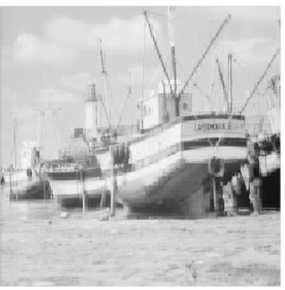

(g)

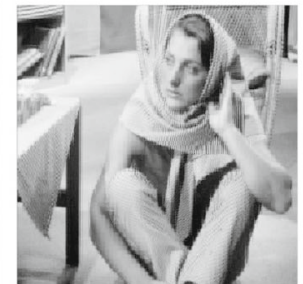

$(\mathrm{k})$

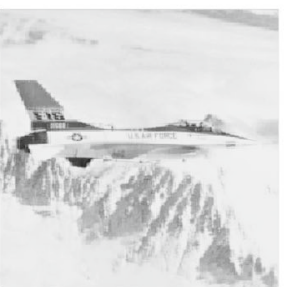

(o)

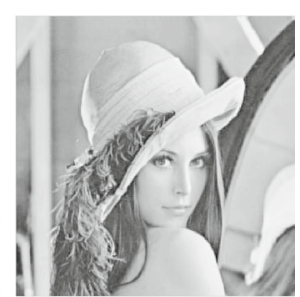

(d)

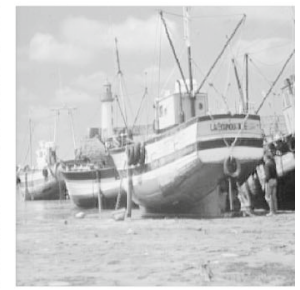

(h)

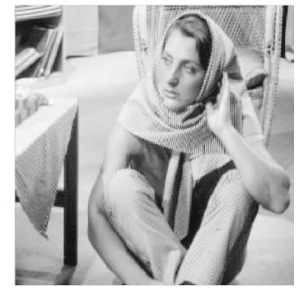

(1)

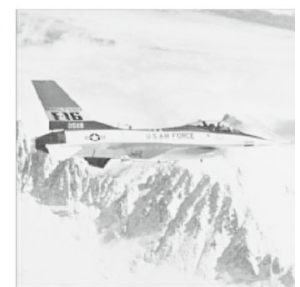

(p)

Fig. 4 Reconstructed images for $r=15$. a T (PSNR=8.572), b T $\mathrm{SFG}$ (PSNR=8.578), $\mathbf{c} C B-2012$ (PSNR=27.500), d BAS-2011 (PSNR=31.271), e T (PSNR=8.231), f T TFG $(P S N R=8.239), \mathbf{g} C B-2012$ (PSNR=25.777), h BAS-2011 (PSNR=28.602), i T (PSNR=9.274), $\mathbf{j} \mathbf{T}_{\mathrm{SFG}}(\mathrm{PSNR}=9.281), \mathbf{k} C B-2012$ (PSNR=24.116), I BAS-2011 (PSNR=25.275), $\mathbf{m}$ T (PSNR=5.711), $\mathbf{n} \mathbf{T}_{\mathrm{SFG}}(\mathrm{PSNR}=5.715), \mathbf{o} C \mathrm{CB}-2012$ (PSNR=26.201) and $\mathbf{p}$ BAS-2011 (PSNR=29.152) 
a constant bitrate encoding based on the retention of $r$ transform-domain coefficients, as suggested originally by Haweel and others [2-11].

Although the authors do not explicitly inform the number of retained coefficients $(r)$ in their computations. Only for high values of $r$ we could obtain similar values. We calculated the PSNR values considering $r=60$. Notice that for such a high value of $r$ data is practically not compressed. This is because only 4 coefficients are discarded, implying a compression rate of only $6.25 \%$. Table 2 shows the results. Additionally, at low compression, most orthogonal transforms tend to behave similarly. However, even under this scenario, the transformation proposed by the authors performed poorly-roughly $10 \mathrm{~dB}$ lower PSNR measurements. Indeed, the pivotal character of a good transform is its behavior in a wide range of compression rates, specially at high compression. For instance, considering the more realistic case of $r=10$, as suggested in [2], we obtain the PSNR values shown in Table 3. Notice that the transformation proposed by the authors exhibits extremely high errors, which are emphasized in bold. We also report that the results linked to the transformations described in [14] and [15] display also acutely poor results as shown in Table 3.
In 'Fig. 3' of their work, the authors show reconstructed compressed images according to the following transformations: $\mathbf{T}_{\mathrm{SFG}}, \mathrm{CB}-2012$, and BAS-2011. All images showed high PSNR values with $\mathbf{T}_{\mathrm{SFG}}$ offering PSNR values greater than $41 \mathrm{~dB}$. We could no reproduce these results. The authors does not detail the employed parameters, in particular the value of $r$. However, for $r=45$, we could obtain comparable PSNR measurements in terms of the traditional DCT approximations. Considering $\mathbf{T}$ or $\mathbf{T}_{\mathrm{SFG}}$ the image degradation is very high, as shown in Fig. 3. For $r=15$, a more realistic value, we obtain the images shown in Fig. 4. Images associated to $\mathbf{T}$ or $\mathbf{T}_{\mathrm{SFG}}$ are severely degraded-roughly 25-30 dB lower than the typical values offered by traditional approximations. These results are evidence that the transformation proposed by the authors is not suitable for image compression.

Authors also show in 'Fig. 4' of their paper a curve relating PSNR measurements of compressed images to the parameter $r$. We could not reproduce their results. Figure 5 shows the curves that we obtained considering the same images as the authors. Our results are compatible to the computations independently found in $[2,4-11]$. The curves associated to $\mathbf{T}$ and $\mathbf{T}_{\mathrm{SFG}}$ indicate

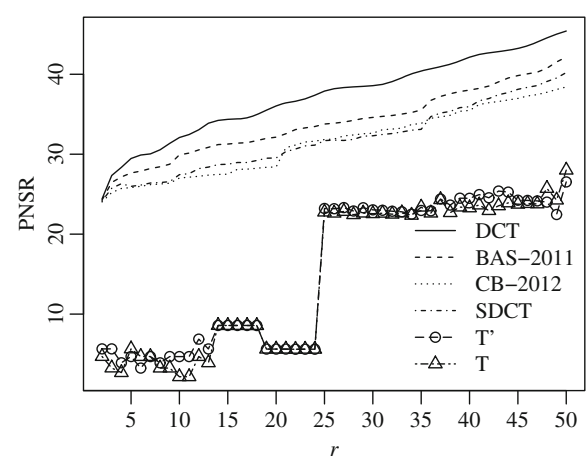

(a)

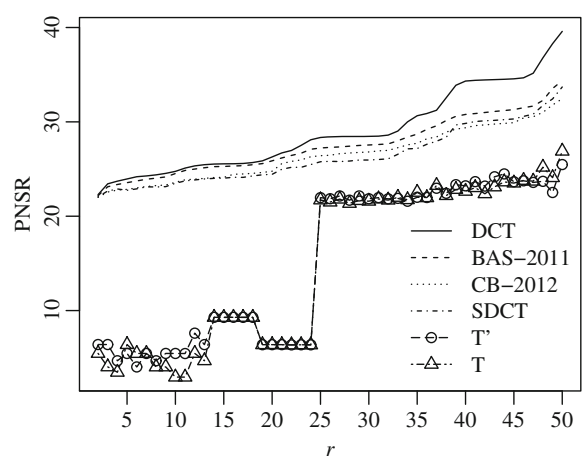

(c)

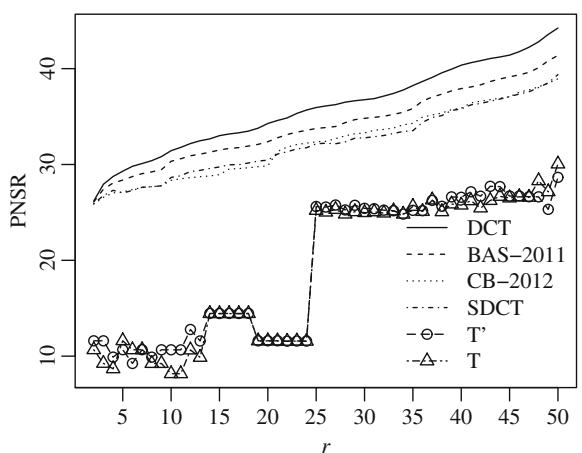

(b)

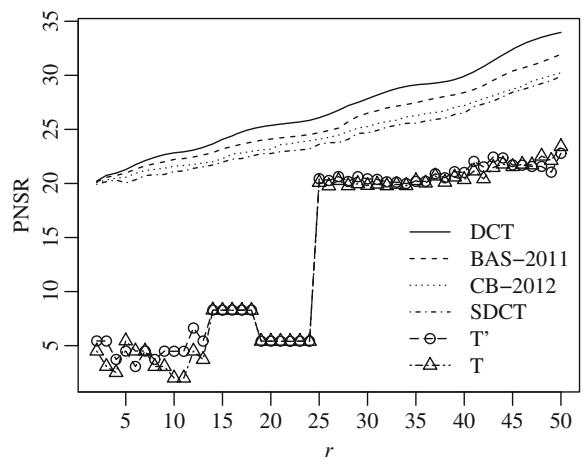

(d)

Fig. 5 PSNR measurements in terms of the number of retained coefficients $r$ for selected standard images. $\mathbf{a}$ Lena, b Cameraman, $\mathbf{c}$ Barbara and d Mandrill 
a significantly lower performance. For $r<25-$ a more realistic scenario-the PSNR loss compared to the traditional transformations is roughly $20 \mathrm{~dB}$. Such evidence points towards the ineffectiveness of $\mathbf{T}$ and $\mathbf{T}_{\mathrm{SFG}}$ for image compression.

\section{Conclusion}

The transformation proposed in [1] performs poorly when compared to archived DCT approximations. The results in [1] could not be reproduced and some corrections are supplied for the benefit of community.

\section{Acknowledgements}

This work was partially supported by CNPq, FACEPE, and FAPERGS.

\section{Competing interests}

The authors declare that they have no competing interests.

\section{Publisher's Note}

Springer Nature remains neutral with regard to jurisdictional claims in published maps and institutional affiliations.

\section{Author details}

${ }^{1}$ Departmento de Estatística, Universidade Federal de Pernambuco, Recife, Brazil. ${ }^{2}$ Departmento de Estatística and LACESM, Universidade Federal de Santa Maria, Santa Maria, Brazil.

Received: 24 November 2015 Accepted: 18 June 2017

Published online: 10 July 2017

\section{References}

1. V Dhandapani, S Ramachandran, Area and power efficient DCT architecture for image compression. EURASIP J. Adv. Signal Process. 180, 1-9 (2014)

2. TI Haweel, A new square wave transform based on the DCT. Signal Process. 82, 2309-2319 (2001)

3. FM Bayer, RJ Cintra, DCT-like transform for image compression requires 14 additions only. Electron. Lett. 48(15), 919-921 (2012). doi:10.1049/el.2012.1148

4. S Bouguezel, MO Ahmad, MNS Swamy, Low-complexity $8 \times 8$ transform for image compression. Electron. Lett. 44(21), 1249-1250 (2008). doi:10.1049/el:20082239

5. S Bouguezel, MO Ahmad, MNS Swamy, in 2nd International Conference on Signals, Circuits and Systems (SCS). A multiplication-free transform for image compression, (2008), pp. 1-4. doi:10.1109/ICSCS.2008.4746898

6. S Bouguezel, MO Ahmad, MNS Swamy, in 2009 International Conference on Microelectronics (ICM). A fast $8 \times 8$ transform for image compression, (2009), pp. 74-77. doi:10.1109/ICM.2009.5418584

7. S Bouguezel, MO Ahmad, MNS Swamy, in Proceedings of 2010 IEEE International Symposium on Circuits and Systems (ISCAS). Image encryption using the reciprocal-orthogonal parametric transform, (2010), pp. 2542-2545. doi:10.1109/ISCAS.2010.5537110

8. S Bouguezel, MO Ahmad, MNS Swamy, in 53rd IEEE International Midwest Symposium on Circuits and Systems (MWSCAS). A novel transform for image compression, (2010), pp. 509-512. doi:10.1109/MWSCAS.2010.5548745

9. S Bouguezel, MO Ahmad, MNS Swamy, in Proceedings of the 2011 IEEE International Symposium on Circuits and Systems. A low-complexity parametric transform for image compression, (2011)

10. S Bouguezel, MO Ahmad, MNS Swamy, Binary discrete cosine and Hartley transforms. IEEE Trans. Circuits Syst. I: Regular Papers. 60(4), 989-1002 (2013)

11. N Brahimi, S Bouguezel, in 7th International Workshop on Systems, Signal Processing and Their Applications (WOSSPA). An efficient fast integer DCT transform for images compression with 16 additions only, (2011), pp. 71-74. doi:10.1109/WOSSPA.2011.5931415

12. V Britanak, P Yip, KR Rao, Discrete cosine and sine transforms. (Academic Press, Oxford, 2007)
13. RJ Cintra, FM Bayer, A DCT approximation for image compression. IEEE Signal Process. Lett. 18(10), 579-582 (2011)

14. D Vaithiyanathan, $R$ Seshasayanan, in Proceeding of the International Conference on Advanced Computing and Communication Systems (ICACCS) Low power DCT architecture for image compression, (Coimbatore, Tamil Nadu, India, 2013), pp. 1-6. doi:10.1109/ICACCS.2013.6938745

15. D Vaithiyanathan, R Seshasayanan, S Anith, K Kunaraj, in Proceeding of the International Conference on Green Computing, Communication and Conservation of Energy (ICGCE 2013). A low-complexity DCT approximation for image compression with 14 additions, (Chennai, Tamil Nadu, India, 2013), pp. 303-307. doi:10.1109/ICGCE.2013.6823450

\section{Submit your manuscript to a SpringerOpen ${ }^{\circ}$ journal and benefit from:}

- Convenient online submission

- Rigorous peer review

Open access: articles freely available online

- High visibility within the field

- Retaining the copyright to your article

Submit your next manuscript at $\$$ springeropen.com 